\title{
Detoxifying carcinogenic polyhalogenated quinones by hydroxamic acids via an unusual double Lossen rearrangement mechanism
}

\author{
Ben-Zhan Zhu'a,b,1, Jun-Ge Zhu', Li Mao ${ }^{\mathrm{a}}$, Balaraman Kalyanaraman', and Guo-Qiang Shan ${ }^{\mathrm{a}}$ \\ aState Key Laboratory of Environmental Chemistry and Ecotoxicology, Research Center for Eco-Environmental Sciences, Chinese Academy of Sciences, \\ Beijing 100085, China; 'binus Pauling Institute, Oregon State University, Corvallis, OR 97331; and 'Department of Biophysics, Medical College of \\ Wisconsin, Milwaukee, WI 53226
}

Edited by Jack Halpern, University of Chicago, Chicago, IL, and approved October 8, 2010 (received for review July 26, 2010)

\begin{abstract}
Hydroxamic acids, which are best-known for their metal-chelating properties in biomedical research, have been found to effectively detoxify the carcinogenic polyhalogenated quinoid metabolites of pentachlorophenol and other persistent organic pollutants. However, the chemical mechanism underlying such detoxication is unclear. Here we show that benzohydroxamic acid (BHA) could dramatically accelerate the conversion of the highly toxic tetrachloro-1, 4-benzoquinone ( $p$-chloranil) to the much less toxic 2 , 5-dichloro-3, 6-dihydroxy-1, 4-benzoquonine (chloranilic acid), with rate accelerations of up to 150,000 -fold. In contrast, no enhancing effect was observed with 0 -methyl BHA. The major reaction product of BHA was isolated and identified as $O$-phenylcarbamyl benzohydroxamate. On the basis of these data and oxygen-18 isotope-labeling studies, we proposed that suicidal nucleophilic attack coupled with an unexpected double Lossen rearrangement reaction was responsible for this remarkable acceleration of the detoxication reaction. This is the first report of an unusually mild and facile Lossen-type rearrangement, which could take place under normal physiological conditions in two consecutive steps. Our findings may have broad biological and environmental implications for future research on hydroxamic acids and polyhalogenated quinoid carcinogens, which are two important classes of compounds of major biomedical and environmental interest.
\end{abstract}

$\mathbf{P}_{\mathrm{i}}^{\mathrm{o}}$ olyhalogenated quinones represent a class of toxicological intermediates that can create a variety of hazardous effects in vivo, including acute hepatoxicity, nephrotoxicity, and carcinogenesis $(1,2)$. Tetrachloro-1, 4-benzoquinone (TCBQ) is one of the major genotoxic and carcinogenic quinoid metabolites of the widely used wood preservative pentachlorophenol (PCP). PCP has been found in at least 313 of the 1,585 National Priorities List sites identified by the US Environmental Protection Agency and classified as a group 2B environmental carcinogen by the International Association for Research on Cancer (3). TCBQ has also been observed as a reactive oxidation intermediate or product in processes used to oxidize or destroy PCP and other polychlorinated persistent organic pollutants (POPs) in various chemical and enzymatic systems (3-7). TCBQ itself has been widely used as a fungicide (Spergon) for treatment of seeds and foliage, and as an oxidizing or dehydrating agent in organic synthesis (often called $p$-chloranil).

Hydroxamic acids have attracted considerable interest recently because of their capacity to inhibit a variety of enzymes such as metalloproteases and lipoxygenase, and transition metalmediated oxidative stress. Some hydroxamates, such as suberoylanilide hydroxamic acid and deferoxamine, have been used clinically for the treatment of cancer or iron-overload diseases (8-11). Much of the activities of these hydroxamic acids were thought to be due to their metal-chelating properties. Deferoxamine is a naturally occurring trihydroxamate siderophore that has been widely used as an iron-chelating drug for the clinical treatment of $\beta$-halassemia $(3,8)$. In our previous work, we found that deferoxamine and other hydroxamic acids, but not other classic iron chelators such as diethylenetriaminepentaacetic acid, provided strong protection against PCP quinoid metabolite-induced cytoand genotoxicity in human fibroblasts $(12,13)$. During our recent studies on metal-independent decomposition of hydroperoxides by halogenated quinones, we showed that these hydroxamic acids could also markedly inhibit TCBQ-mediated hydroperoxide decomposition and hydroxyl/alkoxyl radical formation (14-17). Interestingly, we found that the protection or inhibition by these hydroxamic acids was not due to their iron-chelating properties, but possibly due to their effective scavenging of the reactive tetrachlorosemiquinone radical and (or) their remarkable acceleration of TCBQ hydrolysis to the much less reactive and almost nontoxic 2, 5-dichloro-3, 6-dihydroxy-1, 4-benzoquonine (DDBQ, also called chloranilic acid) (12-14).

It was a great surprise for us to find that hydroxamic acids could markedly accelerate the conversion of TCBQ to DDBQ, because we could not find a reasonable explanation based on our chemical knowledge at the time. Although we first observed the above interesting phenomenon a decade ago (12), its underlying molecular mechanism remained a puzzle. Recently, the reactions between TCBQ and hydroxamic acids were carefully reexamined, and the reaction products were isolated, purified and unambiguously identified by HPLC/MS, NMR, and oxygen-18 isotopelabeling methods. We found, unexpectedly, that a unique double Lossen rearrangement was responsible for this unusual reaction.

\section{Results and Discussion}

Dramatic Acceleration of TCBQ Hydrolysis by Benzohydroxamic Acid. TCBQ has been shown to hydrolyze first to the initial transient monohydroxylation intermediate, trichlorohydroxy-1,4-benzoquinone (TrCBQ-OH), and then very slowly to the final dihydroxylation product, DDBQ, at neutral $\mathrm{pH}(18)$. We found that hydroxamic acids could markedly accelerate this TCBQ hydrolysis process (Note that the meaning for "TCBQ hydrolysis" or "hydrolysis of TCBQ" was specifically defined as "the conversion of TCBQ to TrCBQ-OH or DDBQ"), which could be monitored by increased absorbance at $332 \mathrm{~nm}$. Among the five hydroxamic acids tested, the most effective one was benzohydroxamic acid (BHA) (Fig. 1). Therefore, BHA was chosen as a model hydroxamic acid in the present mechanistic research. In our previous studies $(12,13)$, we found that the initial transient intermediate and final products of TCBQ after reacting with BHA were probably TrCBQ-OH and DDBQ, respectively, and this was confirmed to be the case by electrospray ionization quadrupole

Author contributions: B.-Z.Z. and J.-G.Z. designed research; B.-Z.Z., J.-G.Z., L.M., and G.-Q.S performed research; B.K. contributed new reagents/analytic tools; B.-Z.Z., J.-G.Z., L.M., and G.-Q.S. analyzed data; and B.-Z.Z. and J.-G.Z. wrote the paper.

The authors declare no conflict of interest.

This article is a PNAS Direct Submission.

${ }^{1}$ To whom correspondence should be addressed. E-mail: bzhu@rcees.ac.cn.

This article contains supporting information online at www.pnas.org/lookup/suppl/ doi:10.1073/pnas.1010950107///DCSupplemental. 


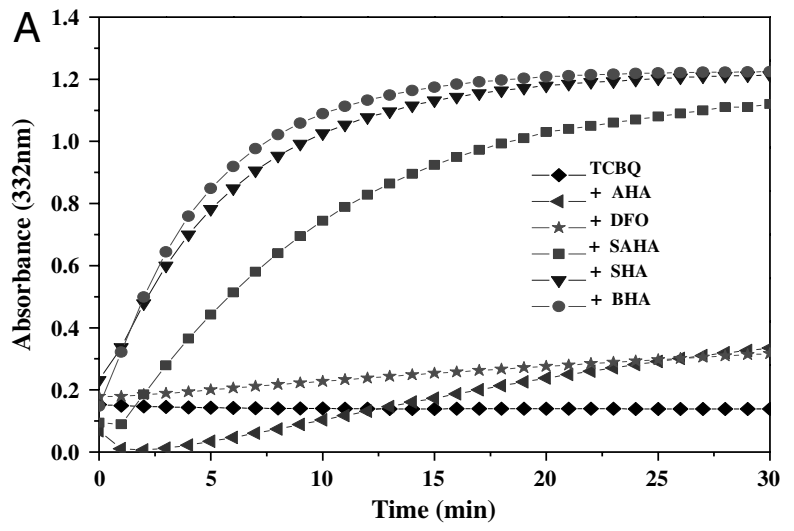

(A)

Fig. 1. (A) The hydrolysis of TCBQ to DDBQ was markedly enhanced by BHA and other hydroxamic acids. All incubation mixtures contained $0.2 \mathrm{mM}$ hydroxamic acids in phosphate buffer $(10 \mathrm{mM}, \mathrm{pH} 7.0)$ at room temperature. The reactions were initiated by the addition of TCBQ $(0.05 \mathrm{mM})$, followed by rapid mixing. DDBQ formation was monitored at $332 \mathrm{~nm}$ for $30 \mathrm{~min}$. AHA: acetohydroxamic acid; DFO: deferoxamine; SAHA: suberoylanilide hydroxamic acid; and SHA: salicylhydroxamic acid. Each point represents the mean of two separate experiments with the SD less than $5 \%$. (B) The chemical structures of hydroxamic acids used in this study.

time-of-flight mass spectrometry (Fig. 2). For details, see Materials and Methods. The extent of formation of TrCBQ-OH and DDBQ was found to be dependent on the molar ratios between BHA and TCBQ: at low molar ratios $(\leq 1)$, TCBQ was converted primarily to TrCBQ-OH; at higher molar ratios $(>1)$, TCBQ could be converted first to TrCBQ-OH and then further to DDBQ (Fig. $2 A$ and $C-F$ ). To test whether BHA could also directly accelerate the slow hydrolysis of TrCBQ-OH to DDBQ, TrCBQ-OH was synthesized according to a published method (16). We found that this was indeed the case (Fig. $2 B$ and $F$ ).

The rate of hydrolysis was found to be also dependent on the molar ratios of BHA/TCBQ and BHA/TrCBQ-OH (Fig. $3 A$ and $B)$ : The higher the molar ratios, the faster the hydrolysis. For example, the half time of hydrolysis of TCBQ $(0.05 \mathrm{mM})$ to DDBQ in the presence of $0.2,0.5,1.0$, and $5.0 \mathrm{mM} \mathrm{BHA}$ was found to be $2.00,0.88,0.45$, and $0.20 \mathrm{~min}$, respectively ( $\mathrm{pH} 7.0$ ). Because it has been shown that the half time for the spontaneous hydrolysis of TCBQ to DDBQ was $21 \mathrm{~d}$ (18), our results demonstrate that BHA could enhance the hydrolysis of TCBQ to DDBQ up to 150,000-fold (TCBQ, $0.05 \mathrm{mM}$; BHA, $5.0 \mathrm{mM}$ ).

Analogous results were observed when TCBQ was substituted with other tetrahalogenated quinones, including tetrabromo- and tetrafluoro-1,4-benzoquinones and their corresponding hydroquinone forms, as well as tetrabromo- and tetrachloro-1,2-benzoquinones. When BHA was substituted with other hydroxamic acids, including salicyl-, suberoylanilide-, and aceto-hydroxamic acids, as well as deferoxamine, similar results were obtained. These findings suggest that this is a general reaction between hydroxamic acids and tetrahalogenated quinoid compounds.

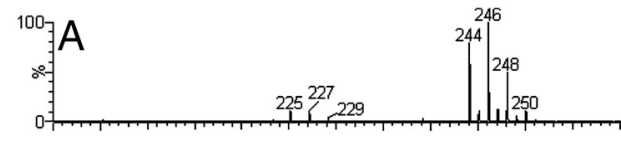

TCBQ

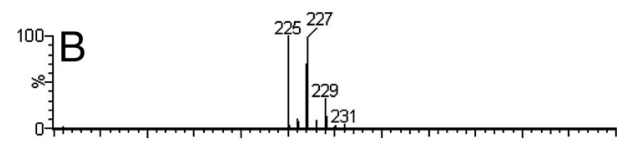

TrCBQ-OH
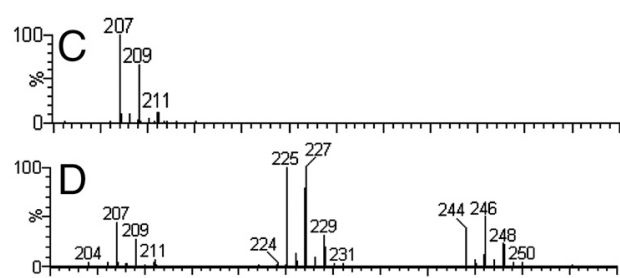

DDBQ

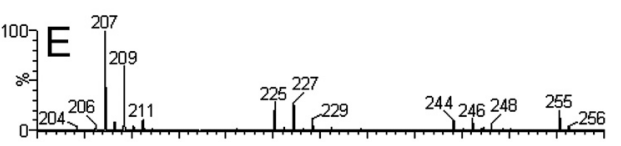

TCBQ/BHA $=1: 4$

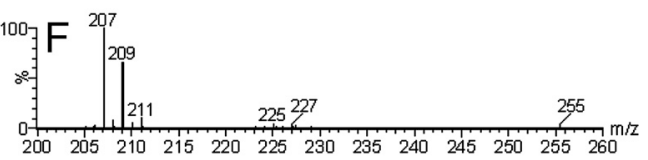

$\operatorname{TrCBQ}-\mathrm{OH} / \mathrm{BHA}=1: 4$

Fig. 2. Mass spectral analysis. The ESI-Q-TOF-MS spectra of TCBQ $(A), \operatorname{TrCBQ}$ $\mathrm{OH}(B), \mathrm{DDBQ}(C), \mathrm{TCBQ}$ with BHA at the ratio of $1: 1(D), 1: 4(E)$, and TrCBQ$\mathrm{OH}$ with $\mathrm{BHA}$ at the ratio of $1: 4(F)$ in $\mathrm{CH}_{3} \mathrm{COONH}_{4}$ buffer $(\mathrm{pH} 7.0,0.1 \mathrm{M})$ at room temperature. TCBQ, $1 \mathrm{mM}$; TrCBQ-OH, $1 \mathrm{mM}$; BHA, 1 or $4 \mathrm{mM}$; MS/MS analysis showed that the peak at $m / z 255$ is the main reaction product $O$-phenylcarbamyl benzohydroxamate (I).

Free Benzohydroxamate Anion Is Essential for the Acceleration of TCBQ Hydrolysis. The rate of BHA-mediated hydrolysis of TCBQ to DDBQ was found to be dependent on the $\mathrm{pH}$ of the buffer. No hydrolysis was observed at $\mathrm{pH} \leq 4$ but as the $\mathrm{pH}$ was increased, the rate of hydrolysis increased progressively (Fig. $3 C$ ). These results suggest that the free benzohydroxamate anion is probably the reactive form of BHA $\left(p K_{a}=8.9\right)$. To further test the above hypothesis, $O$-methyl BHA $\left(\mathrm{Ph}-\mathrm{C}(\mathrm{O})-\mathrm{NH}-\mathrm{O}-\mathrm{CH}_{3}\right)$ was synthesized to block the formation of the free anionic oxygen (19). As anticipated, $O$-methyl BHA had no enhancing effect on TCBQ hydrolysis, even at $\mathrm{pH}$ values as high as 10 (Fig. 3D). The above results clearly demonstrate that the free benzohydroxamate anion is essential for the dramatic acceleration of TCBQ hydrolysis to occur.

Major Reaction Product of BHA Was Identified as $\boldsymbol{0}$-phenylcarbamyl Benzohydroxamate. Because the rate of TCBQ hydrolysis in the presence of $\mathrm{BHA}$ is much faster than that of its spontaneous hydrolysis, it appeared that BHA could catalyze this process. However, we found that BHA was consumed during its reaction with TCBQ, with the concurrent formation of a unique compound with $m / z$ at 255 (Fig. $2 E$ ). This suggested that the hydroxamic acid is not a true catalyst in the TCBQ hydrolysis reaction. To better understand the underlying molecular mechanism for this reaction, the final products of BHA after reacting with TCBQ were then isolated, purified and identified by HPLC-ESI-MS and NMR. Interestingly, $O$-phenylcarbamyl benzohydroxamate (I) was identified as the major reaction product of BHA, whereas $N, N^{\prime}$-diphenylurea (II) as a minor product (For experimental details, see Materials and Methods; for chemical structures of products (I) and (II), see below; $\mathrm{Ar}=$ phenyl group).

Then the question is: How are these products (I and II) formed? It has been shown $(20,21)$ that I could be formed through nucleophilic addition of phenyl isocyanate with a molecule of BHA: 

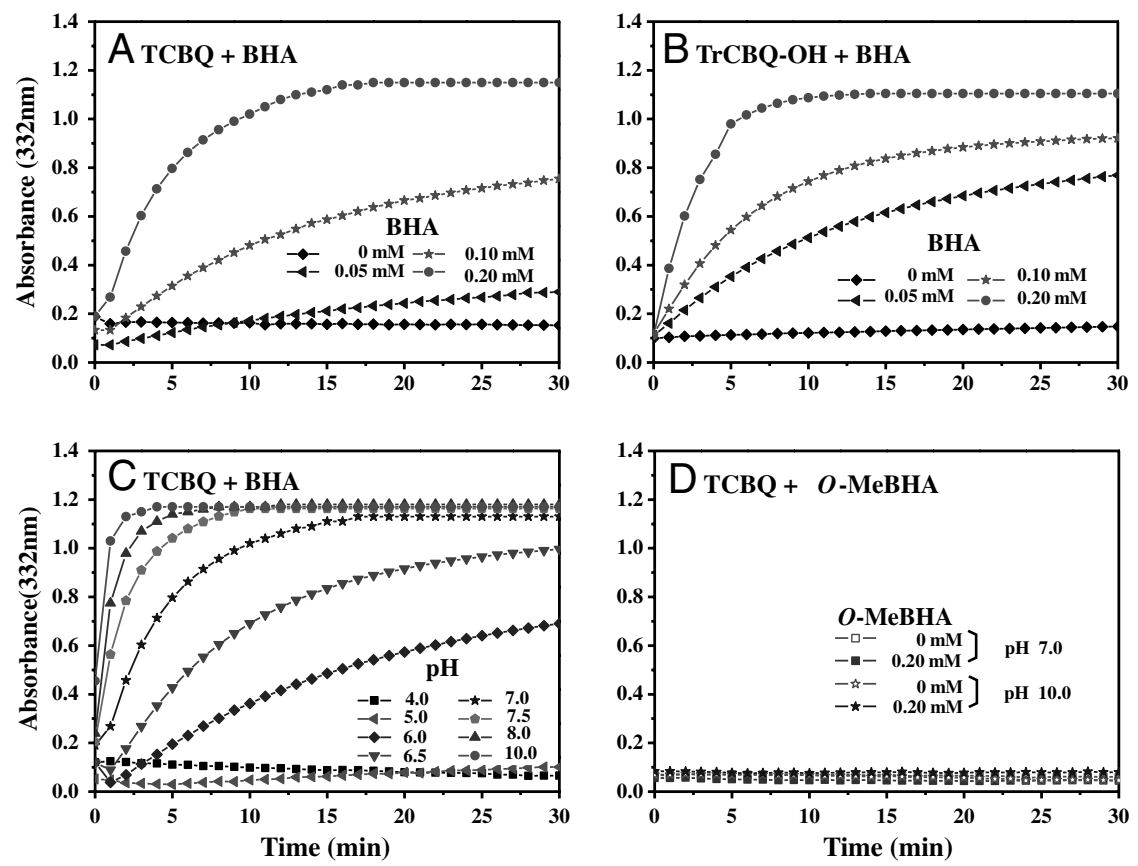

Fig. 3. $B H A$, but not $O-M e B H A$, enhanced the hydrolysis of TCBQ and TrCBQ-OH to DDBQ in a concentration- and $\mathrm{pH}$-dependent manner. $(A) \mathrm{BHA}$ enhanced TCBQ hydrolysis in a concentration-dependent manner. (B) BHA enhanced TrCBQ-OH hydrolysis in a concentration-dependent manner. (C) BHA enhanced TCBQ hydrolysis in a pH-dependent manner; TCBQ, $0.05 \mathrm{mM}$; BHA, $0.20 \mathrm{mM}$. (D) O-MeBHA could not enhance TCBQ hydrolysis. All incubation mixtures contained the indicated concentration of BHA in phosphate buffer ( $10 \mathrm{mM}, \mathrm{pH} 7.0)$ at room temperature. The reactions were initiated by the addition of TCBQ or TrCBQ-OH (0.05 mM), followed by rapid mixing. DDBQ formation was monitored at $332 \mathrm{~nm}$ for $30 \mathrm{~min}$.

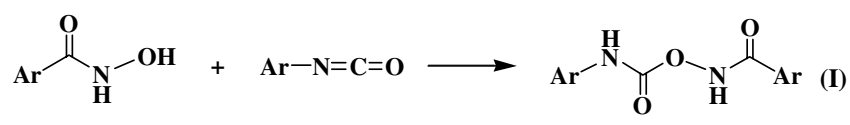

and II could be formed through the hydrolysis of phenyl isocyanate to aniline, followed by reaction of aniline with another molecule of phenyl isocyanate:

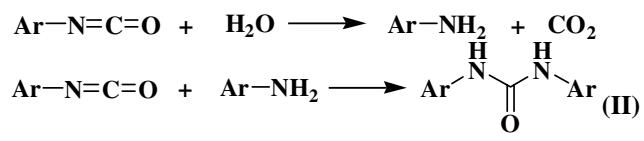

The above analysis strongly suggests that phenyl isocyanate $(\mathrm{Ar}-\mathrm{N}=\mathrm{C}=\mathrm{O})$ should be formed as an initial unstable product during the reaction of BHA and TCBQ.

This leads to a new question: In what way could phenyl isocyanate be produced from BHA? It has been shown that one typical way could be through the classic Lossen rearrangement, a wellknown reaction that describes the transformation of an $O$-activated hydroxamic acid ( $\mathrm{R}-\mathrm{C}(\mathrm{O})-\mathrm{NH}-\mathrm{OX})$ into the corresponding isocyanate $(21,22)$. The rate-limiting step of this reaction is the activation of the hydroxamic acid by various agents (i.e., sulfonyl and benzoyl chloride, etc.; $\left.\mathrm{X}=\mathrm{SO}_{2} \mathrm{R}, \mathrm{C}(\mathrm{O}) \mathrm{R}\right)$ :

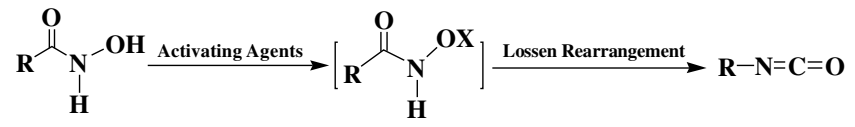

The above analysis suggested that the reaction between BHA and TCBQ might proceed through an analogous, but previously unknown Lossen-type rearrangement pathway.

Molecular Mechanism for the Acceleration of TCBQ Hydrolysis by BHA: Suicidal Nucleophilic Attack Coupled with an Unusual Double Lossen Rearrangement. Based on the above experimental results and earlier research on Lossen rearrangement (20-23), as well as the fact that the benzohydroxamate anion is a particularly effective $\alpha$-nucleophile (20), a unique mechanism for BHA-accelerated TCBQ hydrolysis was proposed (Scheme 1): According to the mechanism, a nucleophilic reaction takes place between the benzohydroxamate anion $\left(\mathrm{ArC}(\mathrm{O})-\mathrm{NH}^{-} \mathrm{O}^{-}\right)$and $\mathrm{TCBQ}$, first forming an unstable transient intermediate $\mathrm{ArC}(\mathrm{O})$ - $\mathrm{NH}-\mathrm{O}$-trichloro- 1,4-benzoquinone. Following loss of a proton from nitrogen to form the anionic $\operatorname{ArC}(\mathrm{O})-\mathrm{N}^{-}$-O-trichloro-1,4-benzoquinone intermediate, a spontaneous Lossen-type rearrangement leads to the formation of $\mathrm{TrCBQ}^{-} \mathrm{O}^{-}$(at low $\mathrm{BHA} / \mathrm{TCBQ}$ molar ratios) and phenyl isocyanate. When $\mathrm{BHA}$ is in excess, TrCBQ-Ofurther reacts with $\mathrm{BHA}$, through a similar reaction intermediate, and a second-step spontaneous Lossen-type rearrangement reaction yields DDBQ and another molecule of phenyl isocyanate. As mentioned above, phenyl isocyanate could react with another molecule of BHA to yield the major reaction product $\mathbf{I}$.

The second equivalent of benzohydroxamate anion is shown in Scheme 1 displacing the chloride ion para to the oxyanion resulting from the first addition of benzohydroxamate anion. Why this regioselectivity is favored over meta or ortho displacement? There are probably two major reasons for this favored regioselectivity: One is due to the steric hindrance to form the chloroquinone-BHA intermediate. Para displacement of the chloride ion should have less steric hindrance than meta or ortho displacement; another is due to the stability of the thus-formed end products: 2, 5-dichloro-3, 6-dihydroxy-1, 4-benzoquinone (DDBQ, chloranilic acid) should be much more stable than 2 , 3-dichloro-5, 6-dihydroxy- or 2, 6-dichloro-3,5-dihydroxy-1, 4benzoquinone.

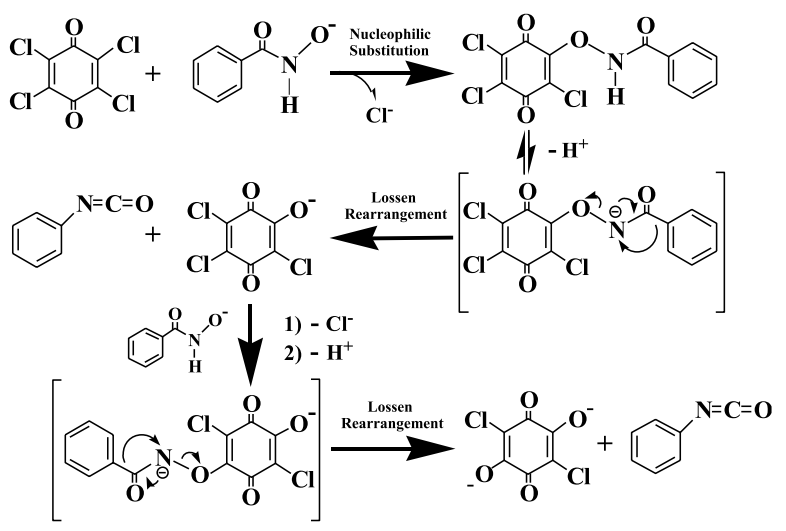

Scheme 1. Proposed mechanism for the dramatic acceleration of TCBQ hydrolysis by BHA: Suicidal nucleophilic attack coupled with an unusual double Lossen rearrangement. 
If the above proposed double Lossen rearrangement mechanism is correct, then we expect that the source and origin of the oxygen atom in DDBQ and product $\mathbf{I}$ from the reaction of TCBQ (or TrCBQ-OH) with BHA should be from the hydroxyl group of $\mathrm{BHA}$, not from water. To test whether this is the case, TCBQ (or TrCBQ-OH) was incubated with BHA in buffer solution prepared with oxygen-18-labeled $\mathrm{H}_{2} \mathrm{O}\left(\left[{ }^{18} \mathrm{O}\right]-\mathrm{H}_{2} \mathrm{O}\right)$ as solvent. If water is the source of the oxygen atom in any of the products, the mass spectra of the molecular ion region of DDBQ and product I, obtained with unlabeled and labeled $\mathrm{H}_{2} \mathrm{O}$, should show the shift of the molecular ion isotope cluster peaks of the unlabeled compounds with 2 or 4 mass units, as could be expected for the incorporation of ${ }^{18} \mathrm{O}$. However, no ${ }^{18} \mathrm{O}$ was found to be incorporated in any of these products, as shown in Fig. S1 (see SI Text). These isotope-labeling data provide strong experimental evidence to further support the above proposed mechanism.

Why this Lossen Rearrangement Reaction Is So Unusual. It has been well established that the activation of the oxygen atom of hydroxamic acids is essential for the Lossen rearrangement to take place. Therefore, the Lossen rearrangement is customarily carried out by using prepared $O$-activated hydroxamic acid derivatives or by in situ activation of free hydroxamic acids with various activating agents such as polyphosphoric acid and carbodiimides (20-23). To our knowledge, this is the only report demonstrating that TCBQ and other tetrahalogenated quinones could serve as a unique class of agents for the activation of free hydroxamic acids, forming unstable $O$-trihaloquinonated hydroxamic acids, leading spontaneously to two consecutive Lossen-type rearrangements.

Most of the previously reported Lossen rearrangement reactions take place only under alkaline conditions and/or through heating to a requisite temperature (20-23). In the present study, we found that the reaction between BHA and TCBQ could occur at room temperature and under neutral or even weakly acidic $\mathrm{pH}$. This is possibly due to the unusually rapid and facile rearrangement of the postulated reaction intermediate, the $O$-trichloroquinonated BHA. It has been shown that the rate-limiting step in the Lossen rearrangement is the activation of the hydroxamic acid, and the rate of the rearrangement is directly proportional to the relative acidity of the conjugate acid of the anionic leaving group $(21,22)$. Due to the strong acidity of TrCBQ-OH $\left(p K_{a}: 1.10\right)$ and DDBQ $\left(p K_{a} 1: 0.58 ; p K_{a} 2: 2.72\right)(7,18)$, which are the conjugate acids of the anionic leaving groups in the present study, it is expected that the rate of the rearrangement of the postulated reaction intermediates should be very fast. Once formed, they rearrange extremely rapidly at room temperature and under neutral $\mathrm{pH}$. This could explain why BHA and other hydroxamic acids could dramatically accelerate the hydrolysis of TCBQ (or TrCBQ-OH) to DDBQ under physiological conditions of $\mathrm{pH}$ and temperature. This could also explain why, unlike most $O$-acyl hydroxamic acids, the postulated reaction intermediate could not be isolated and identified in the present study.

Potential Biological and Environmental Implications. We found that this unusual double rearrangement reaction mechanism is not only limited to TCBQ and benzohydroxamic acid, but it is also a general mechanism for all tetrahalogented quinonoid compounds and hydroxamic acids. Therefore, our findings may have interesting biological and environmental implications: Many widely used polyhalogenated aromatic compounds (which are considered POPs and probable human carcinogens) including polyhalogenated phenols (such as the ubiquitous PCP and the brominated flame-retardant 3,3',5,5'-tetrabromobisphenol A), Agent Orange, hexachlorobenzene, hexachlorocyclohexane isomers, and polychlorinated biphenyls, can be metabolized in vivo (1-3, 24-26), or dehalogenated chemically and enzymatically (4-6), to their corresponding quinones. Polychlorinated quinoid compounds were also found in discharges from pulp and paper mills (3). More recently, several polyhalogenated quinones, which are suspected bladder carcinogens, were identified as unique chlorination disinfection byproducts in drinking water (27). These polyhalogenated quinones not only cause oxidative damage to DNA and other macromolecules, but also form protein and DNA adducts both in vitro and in vivo (1-3, 24-26). Thus, these molecules are potential mammalian carcinogens, which render their destruction or remediation under mild conditions of critical importance.

Of particular interest in this regard is the fact that two hydroxamic acids are already approved for clinical applications, deferoxamine for iron overload and suberoylanilide hydroxyamic acid (Vorinostat), recently approved for cutaneous T-cell lymphoma (9-11). As demonstrated in the present and our previous studies (12-17), these compounds, in addition to BHA might be especially suited for detoxication of polyhalogenated quinones. It is worth noting that hydroxamic acids can also efficiently inhibit hexachlorobenzene-induced porphyria (28), and detoxify chemical warfare agents such as the nerve gases Sarin and Soman (which are fluorinated organophosphonates) $(21,22,29)$, as well as nitrogen mustard (30) in animal models, possibly through an analogous Lossen rearrangement mechanism. Therefore suicidal nucleophilic attack coupled with spontaneous Lossen rearrangement may serve as a general, but previously unrecognized detoxication mechanism for the widely-used hydroxamic acids. Therefore, our findings may have broad chemical, biological, and environmental implications for future research on polyhalogenated aromatic pollutants and hydroxamic acids, which are two important classes of compounds of major environmental and biomedical concern that have been attracting the attention of both academic researchers, and the broader general public. Further research is needed to investigate whether hydroxamic acids could be used safely and effectively as prophylactics for the prevention or treatment of human diseases such as liver and bladder cancer associated with the toxicity of polyhalogenated quinoid carcinogens.

\section{Materials and Methods}

Chemicals. Tetrachloro-1,4-benzoquinone (TCBQ; tetrachloro- $p$-benzoquinone or $p$-chloranil) and other halogenated quinones were purchased from Fluka. Suberoylanilide hydroxamic acid (SAHA) was bought from Melone Pharmaceutical Co., Ltd. The oxygen-18-enriched $\mathrm{H}_{2} \mathrm{O}\left(90 \%\right.$, $\left.\left[{ }^{18} \mathrm{O}\right]-\mathrm{H}_{2} \mathrm{O}\right)$ was purchased from J\&K. Benzohydroxamic acid (BHA), salicylhydroxamic acid (SHA), acetohydroxamic acid (AHA), deferoxamine (DFO) and all other chemicals were purchased from Sigma. The chemicals were used as received without further purification.

Spectral Analysis. The interactions between TCBQ and hydroxamic aicds were monitored by a UV-visible spectrophotometer (DU-800, Beckman Coulter), using Chelex-treated phosphate buffer $(10 \mathrm{mM}, \mathrm{pH} 7.0)$ at room temperature. The formation of DDBQ was followed at $332 \mathrm{~nm}$. IR spectra were recorded on a FTS-40 spectrophotometer in $\mathrm{KBr}$. ${ }^{1} \mathrm{H}$ NMR spectra were acquired on a Bruker DPX-400 spectrometer using $\left(\mathrm{CH}_{3}\right)_{4} \mathrm{Si}$, tetramethylsilane as internal standard and $\mathrm{CDCl}_{3}, \mathrm{DMSO}-d_{6}$ as solvent.

Identification of the Reaction Products of TCBQ. The final reaction products between TCBQ and BHA were identified by electrospray ionization quadrupole time-of-flight mass spectrometry (ESI-Q-TOF-MS) (15-17). The mass spectrum of TCBQ in $0.1 \mathrm{M} \mathrm{CH}_{3} \mathrm{COONH}_{4}$ buffer ( $\mathrm{pH} \mathrm{7.0)}$ was characterized by a four-chlorine isotope cluster at $m / z 246$ and traces of a three-chlorine isotope cluster at $m / z$ 227. The addition of BHA $(0.5 \mathrm{mM})$ to TCBQ $(0.5 \mathrm{mM})$ led to partial disappearance of the molecular ion peak clusters at $m / z 246$ and a remarkable increase in the peak clusters at $m / z 227$. These results indicate that one of the major reaction products between BHA and TCBQ at low molar ratios was probably the ionic form of $\operatorname{TrCBQ}-\mathrm{OH}$ (peak clusters at $m / z 227$ ). This was further confirmed by comparison with authentic TrCBQ-OH synthesized according to a published method (16), which showed the same ESI-MS profile and the same retention time on HPLC. When BHA was in excess $(2.0 \mathrm{mM})$, the addition of BHA to TCBQ $(0.5 \mathrm{mM})$ led to almost complete disappearance of the molecular ion peak clusters at $m / z 246$ and $m / z 227$, and a dramatic increase in the peak clusters at $m / z 207$. These results indicate that 
the major reaction product between BHA and TCBQ at high molar ratios was probably the ionic form of DDBQ (peak clusters at $m / z$ 207). This was further confirmed by comparison with authentic DDBQ, which showed the same ESI-MS profile and the same retention time on HPLC.

Isolation, Purification, and Identification of the Reaction Products of BHA. O-phenylcarbamyl benzohydroxamate (I). When the ratio of BHA and TCBQ was low, no precipitate was observed during the reaction of TCBQ with BHA. With increasing BHA/TCBQ ratios, especially when the TCBQ concentration was high, a precipitate appeared in the reaction mixture. The mixture was then filtered and the solid was washed with cold water. After further separation and purification by silica gel (ethyl acetate: petroleum ether = $1: 1.5)$, the compounds were then recrystallized by methylene chloride. Two reaction products were obtained. The major product was a white sheet solid, which decomposed at $232-233^{\circ} \mathrm{C}$. The purity and the identity of this compound were assessed by HPLC and ESI-MS analysis. Only one peak was observed in the HPLC analysis, and an ion peak was evident at $m / z 255$ $\left[(\mathrm{M}-\mathrm{H})^{-}\right]$for ESI-MS analysis. Its chemical structure was further characterized by IR, ${ }^{1} \mathrm{H}$ - and ${ }^{13} \mathrm{C}-\mathrm{NMR}$. IR ( $\left.\mathrm{KBr}\right) v\left(\mathrm{~cm}^{-1}\right): 3252(\mathrm{~N}-\mathrm{H}), 3196(\mathrm{~N}-\mathrm{H}), 1739$ $(\mathrm{O}-\mathrm{C}=\mathrm{O}), 1648(\mathrm{NH}-\mathrm{C}=\mathrm{O}), 1601,1558,1523,1502(\mathrm{Ar}-\mathrm{H}), 1022(\mathrm{C}-\mathrm{O}-\mathrm{C})$ ${ }^{1} \mathrm{H}-\mathrm{NMR}\left(400 \mathrm{MHz}_{1} \mathrm{CDCl}_{3}\right)$ 8: $11.26(\mathrm{~s}, 1 \mathrm{H}, \mathrm{NH}, \mathrm{H}-\mathrm{N}-\mathrm{O}), 9.34(\mathrm{~s}, 1 \mathrm{H}, \mathrm{NH}$, $\mathrm{H}-\mathrm{N}-\mathrm{C}), 7.50-8.10\left(\mathrm{~m}, 5 \mathrm{H},-\mathrm{C}_{6} \mathrm{H}_{5}\right), 7.00-7.50\left(\mathrm{~m}, 5 \mathrm{H}, \mathrm{C}_{6} \mathrm{H}_{5}\right) ;{ }^{13} \mathrm{C}-\mathrm{NMR}$ $\left(100 \mathrm{MHz}, \mathrm{CDCl}_{3}\right) \delta: 206.8(\mathrm{O}-\mathrm{C}=0), 153.8(\mathrm{~N}-\mathrm{C}=\mathrm{O}), 139.7,133.6,130.3$, $130.0,128.8,124.7,120.0(\operatorname{ArC})$. Based on the above experimental data, this compound was identified as $\mathrm{O}$-phenylcarbamyl benzohydroxamate (I).

$\boldsymbol{N}, \boldsymbol{N}^{\prime}$-Diphenylurea (II). The minor product was a white powder with m. p. 238$240^{\circ} \mathrm{C}$. Only one peak was observed in the HPLC analysis, and an ion peak was

1. Bolton JL, Trush MA, Penning TM, Dryhurst G, Monks TJ (2000) Role of quinones in toxicology. Chem Res Toxicol 13:135-160.

2. Song Y, Wagner BA, Witmer JR, Lehmler HJ, Buettner GR (2009) Nonenzymatic displacement of chlorine and formation of free radicals upon the reaction of glutathione with PCB quinones. Proc Natl Acad Sci USA 106:9725-9730.

3. Zhu B-Z, Shan G-Q (2009) Potential mechanism for pentachlorophenol-induced carcinogenicity: A novel mechanism for metal-independent production of hydroxyl radicals. Chem Res Toxicol 22:969-977.

4. Meunier B (2002) Catalytic degradation of chlorinated phenols. Science 296:270-271.

5. Gupta SS, et al. (2002) Rapid total destruction of chlorophenols by activated hydrogen peroxide. Science 296:326-328.

6. Sorokin A, Seris JL, Meunier B (1995) Efficient oxidative dechlorination and aromatic ring-cleavage of chlorinated phenols catalyzed by iron sulfophthalocyanine. Science 268:1163-1166.

7. Manderville RA, Pfohl-Leszkowicz A (2006) Advances Molecular Toxicology, ed JC Fishbein (Elsevier, Amsterdam), pp 85-138.

8. Yu Y, Wong J, Lovejoy DB, Kalinowski DS, Richardson DR (2006) Chelators at the cancer coalface: Desferrioxamine to triapine and beyond. Clin Cancer Res 12:6876-6883.

9. Marks PA, Breslow R (2007) Dimethyl sulfoxide to vorinostat: Development of this histone deacetylase inhibitor as an anticancer drug. Nat Biotechnol 25:84-90.

10. Li N, et al. (2008) HDAC inhibitor reduces cytokine storm and facilitates induction of chimerism that reverses lupus in anti-CD3 conditioning regimen. Proc Natl Acad Sc USA 105:4796-4801.

11. Choudhary C, et al. (2009) Lysine acetylation targets protein complexes and co-regulates major cellular functions. Science 325:834-840.

12. Zhu B-Z, HarEl R, Kitrossky N, Chevion M (1998) New modes of action of desferrioxamine: Scavenging of semiquinone radical and stimulation of hydrolysis of tetrachlorohydroquinone. Free Radical Bio Med 24:360-369.

13. Witte l, et al. (2000) Protection by desferrioxamine and other hydroxamic acids against tetrachlorohydroquinone-induced cyto- and genotoxicity in human fibroblasts. Free Radical Bio Med 28:693-700.

14. Zhu B-Z, Zhao H-T, Kalyanaraman B, Frei B (2002) Metal-independent production of hydroxyl radicals by halogenated quinones and hydrogen peroxide: An ESR spin trapping study. Free Radical Bio Med 32:465-473.

15. Zhu B-Z, Kalyanaraman B, Jiang G-B (2007) Molecular mechanism for metal-independent production of hydroxyl radicals by hydrogen peroxide and halogenated quinones. Proc Natl Acad Sci USA 104:17575-17578. evident at $m / z 211\left[(\mathrm{M}-\mathrm{H})^{-}\right]$for ESI-MS analysis. Its chemical structure was further characterized by IR, ${ }^{1} \mathrm{H}$ - and ${ }^{13} \mathrm{C}-\mathrm{NMR}$. IR $(\mathrm{KBr}) v\left(\mathrm{~cm}^{-1}\right): 3327(\mathrm{~N}-\mathrm{H})$, $3289(\mathrm{~N}-\mathrm{H}), 1650(\mathrm{C}=\mathrm{O}), 1597,1498(\mathrm{Ar}-\mathrm{H}) ;{ }^{1} \mathrm{H}-\mathrm{NMR}\left(400 \mathrm{MHz}, \mathrm{CDCl}_{3}\right) \delta: 8.67$ (s, 1H, NH, H-N-C), 6.95-7.53 (t, 5H, $\left.-\mathrm{C}_{6} \mathrm{H}_{5}\right) ;{ }^{13} \mathrm{C}-\mathrm{NMR}\left(100 \mathrm{MHz}, \mathrm{CDCl}_{3}\right) \delta: 152.5$ (O-C=O), 139.7, 128.7, 121.7, 118.2 (ArC). Based on the above experimental data, this compound was identified as $N, N^{\prime}$-diphenylurea (II).

Synthesis of the Reaction Intermediate Trichlorohydroxy-1,4-benzoquinone (TrCBQ-OH). TrCBQ-OH was synthesized as described in the literature by dissolving solid TCBQ (30 mg) in an ice-cold $2 \mathrm{M}$ sodium hydroxide solution (16).

Synthesis of 0-Methyl Benzohydroxamic Acid (0-Methyl BHA). O-Methyl BHA was synthesized as described by Johnson et al. (19).

Oxygen-18 Isotope-Labeling Experiment. To investigate the source and origin of the oxygen atom inserted into DDBQ and product I, formed from the reaction between BHA and TCBQ (or TrCBQ-OH), $1.0 \mathrm{mM} \mathrm{TCBQ}$ (or TrCBQ-OH) was incubated with $B \mathrm{HA}$ in oxygen-18-enriched $\mathrm{H}_{2} \mathrm{O}\left(90 \%,\left[{ }^{18} \mathrm{O}\right]-\mathrm{H}_{2} \mathrm{O}\right)$ in a final volume of $0.1 \mathrm{~mL}$ of $\mathrm{CH}_{3} \mathrm{COONH}_{4}$ buffer $(0.1 \mathrm{M}, \mathrm{pH} 7.0)$ containing $10 \%$ DMSO. A control experiment with unlabeled $\mathrm{H}_{2} \mathrm{O}$ was also performed. After $1.0 \mathrm{~min}$ of incubation, the samples were analyzed by ESI-Q-TOF-MS as described above.

ACKNOWLEDGMENTS. This work was supported by Project 973 Grant 2008CB418106; Hundred-Talent Project, CAS; NSFC Grants 20925724 20777080, 20877081, 20890112, and 20921063; Golda Meir Fellowships from The Hebrew University of Jerusalem; and National Institutes of Health Grants ES11497, RR01008, and ES00210.

16. Zhu B-Z, et al. (2007) Mechanism of metal-independent decomposition of organic hy droperoxides and formation of alkoxyl radicals by halogenated quinones. Proc Natl Acad Sci USA 104:3698-3702.

17. Zhu B-Z, et al. (2009) Metal-independent decomposition of hydroperoxides by halogenated quinones: Detection and identification of a quinone ketoxy radical. Proc Natl Acad Sci USA 106:11466-11471.

18. Sarr DH, Kazunga C, Charles MJ, Pavlovich JG, Aitken MD (1995) Decomposition of telrachloro-1,4-benzoquinone (P-chloranil) in aqueous solution. Environ Sci Technol 29:2735-2740.

19. Johnson JE, Riesgo EC, Jano I (1996) Mechanism of hydrolysis of (Z)- and (E)-O-methylbenzohydroximoyl chloride. J Org Chem 61:45-50.

20. Orth ES, et al. (2009) Suicide nucleophilic attack: Reactions of benzohydroxamate anion with bis(2,4-dinitrophenyl) phosphate. J Org Chem 74:5011-5016.

21. Bauer L, Exner $O$ (1974) Chemistry of hydroxamic acids and $\mathrm{N}$-hydroxyimides. Angew Chem Int Ed Engl 13:376-384.

22. Pereira MMA, Santos PP (2009) The Chemistry of Hydroxylamines, Oximes, and Hydroxamic Acids, eds Z Rappoport and JF Liebman (Wiley, New York), pp 343-499.

23. Dube $P$, et al. (2009) Carbonyldiimidazole-mediated Lossen rearrangement. Org Lett 11:5622-5625

24. Chignell CF, et al. (2008) EPR studies of in vivo radical production by 3,3',5,5'-tetrabromobisphenol A (TBBPA) in the Sprague-Dawley rat. Toxicol Appl Pharmacol 230:17-22.

25. Teuten EL, Xu L, Reddy CM (2005) Two abundant bioaccumulated halogenated compounds are natural products. Science 307:917-920.

26. Kelly BC, Ikonomou MG, Blair JD, Morin AE, and Gobas FA (2007) Food web-specific biomagnification of persistent organic pollutants. Science 317:236-239.

27. Zhao YL, Qin F, Boyd JM, Anichina J, Li XF (2010) Characterization and determination of chloro- and bromo-benzoquinones as new chlorination disinfection byproducts in drinking water. Anal Chem 82:4599-4605.

28. Wainstock de Calmanovici R, Billi SC, Aldonatti CA, San Martín de Viale LC (1986) Effect of desferrioxamine on the development of hexachlorobenzene-induced porphyria. Biochem Pharmacol 35:2399-2405.

29. Bromberg L, et al. (2009) Degradation of chemical warfare agents by reactive polymers. Ind Eng Chem Res 48:1650-1659.

30. Morad $Y$, et al. (2005) Treatment of ocular tissues exposed to nitrogen mustard: Beneficial effect of zinc desferrioxamine combined with steroids. Invest Ophth Vis Sci 46:1640-1646. 